\title{
Two-Level Atom-Field Interaction: Exact Master Equations for Non-Markovian Dynamics, Decoherence and Relaxation
}

\author{
Charis Anastopoulos * and B. L. Hu ${ }^{\dagger}$ \\ Department of Physics, University of Maryland, College Park, Maryland 20742 \\ (umdpp 9\%-129, April 9, 1999)
}

\begin{abstract}
We perform a first- principles derivation of the general master equation to study the nonMarkovian dynamics of a two-level atom (2LA) interacting with an electromagnetic field (EMF). We use the influence functional method which can incorporate the full backreaction of the field on the atom, while adopting Grassmannian variables for the $2 \mathrm{LA}$ and the coherent state representation for the EMF. We find exact master equations for the cases of a free quantum field and a cavity field in the vacuum. In response to the search for mechanisms to preserve maximal coherence in quantum computations in ion trap prototypes, we apply these equations to analyse the decoherence of a $2 \mathrm{LA}$ in an EMF, and fine that decoherence time is close to relaxation time. This is at variance to the claims by authors who studied the same system but used a different coupling model. We explain the source of difference and argue that, contrary to common belief, the EMF when resonantly coupled to an atom does not decohere it as efficiently as a bath does on a quantum Brownian particle. The master-equations for non-Markovian dynamics derived here is expected to be useful for exploring new regimes of $2 \mathrm{LA}-\mathrm{EMF}$ interaction, which is becoming physically important experimentally.
\end{abstract}

*ca81@umail.umd.edu

†hub@physics.umd.edu 


\section{INTRODUCTION}

A two-level system (2LS) interacting with a quantum field - electromagnetic field (EMF) in particular - has proven to be a very useful model for a wide range of problems from atomic-optical [1] 기 and condensed matter [8,9] processes to quantum computation [10]. For the latter application stringent limits in maintaining the coherence of the the 2LS (called qubits) are required. This prompted us to revisit the theoretical structure of the 2LS model, paying special attention to its coherence properties. Treatment of spontaneous emission and relaxation are standard textbook topics, whereas decoherence and dissipation, especially in the context of quantum computation, are the focus of more recent investigations 11 18].

Because of the familiarity of the model (see II.A) and its theoretical and practical values, we do not need to emphasize the general motivation, but can go right to the point about the aim and results of this paper. The description of this system generally comprises of two parts: 1) Spontaneous emission in the 2LS, and 2) Decoherence due to the interaction of 2LS with the EM field, treated as a bath. The first part allows little room for disagreement, as it can be obtained from elementary calculations. The second part on decoherence is more subtle.

Environment-induced decoherence 19] has been studied extensively in recent years primarily based on models of quantum Brownian motion (QBM) 20 26] for the interaction of a simple harmonic oscillator (Brownian particle) with a harmonic oscillator bath (HOB) at a finite temperature, leading to a reasonably good understanding of its characteristic features. Decoherence of a 2LS in an EM field has been studied by a number of authors, notably [11, 12, 16, and their dissipative and decoherent behavior are reported to be similar to that of a QBM in a harmonic oscillator bath. The progression in three stages - quiescent, vacuum fluctuation-dominated and thermal fluctuation-dominated, separated by the cutoff frequency and the thermal de Broglie frequency (wavelength) - are indeed characteristic of the QBM results [21,23 25,27,28].

Our findings, in contrast, are in stark disagreement from that reported in the literature. We work with the standard 2LS-EMF model [1] and obtain an exact master equation for depicting non-Markovian dynamics. Solution of this equation for the reduced density matrix of the 2LS shows that the decoherence rate is close to the relaxation rate. This is in first appearance rather couter-intuitive, and different from all previous findings. Upon careful deliberation we realize that the 'intuition' researchers (including us at the start) have acquired for dissipation and decoherence are based on the QBM model which influenced the choice of model in the investigation of decoherence for a 2LS. However, we find that such a commonly invoked intuition for QBM in a HOB fails to apply to that of a two-level atom (2LA) interacting with an electromagnetic field (EMF) with the commonly assumed type of resonance coupling in quantum optics.

\section{Decoherence in $Q B M$}

Physically, when we say that decoherence of the system of a Brownian oscillator proceeds in a very short time as it is brought in contact with an environment, a HOB at some temperture, we are usually conjuring a model with bilinear [20] (or polynomial [24) coupling of the oscillator-bath coordinates, and a ohmic or subohmic spectral function 21] in the bath. Intuitively, the bath needs to have many degrees of freedom, preferably acting independently of each other so incooperatively that the phase information in the system will be dispersed to the largest extent amongst the many bath degrees of freedom and affords little chance or takes inordinately long time to be revived or reconstituted (recoherence [29]). The opposite picture (of very long decoherence time) is exemplified by two coupled subsystems where no coarse-graining is introduced, or for system-environment couplings which maintain some high level of coherence, or for an environment whose degrees of freedom have long correlation times like in a zero temperature, supraohmic bath. The case of a (spin) particle or (plasma) wave interacting with an averaged (collective) variable from the environment, such as the mean field, showing Landau damping in Vlasov dynamics is another example [30,31. Just as in the spin echo phenomena (e.g, Chap. 3 迎), the basic physics in this case is not dissipation in the Boltzmann sense, but statistical mixing [32]. We will see that this example is of more physical relevance to our problem than the QBM.

\section{Coherence in the $2 L S$}

For the 2LA-EMF system, one clear distinction between an EM field as an environment and a system of harmonic oscillators as bath is that the field (coupled to a detector) has an intrinsic spectral density function, not to be chosen arbitrarily. For example, it has been shown [26] that a conformal scalar field in two dimensions coupled to a monopole detector has an Ohmic character while in four dimensions it is supraohmic. Barone and Caldeira [33] showed that the spectral density function for EM fields with momentum coupling to an oscillator detector is supraohmic. These density functions would show very different decoherence behavior from the high temperature Ohmic HOB used in many discussions of decoherence, the latter case is what the general folklore is based on. But the most important 
distinction from QBM is that the 2LA couples with the EMF in the discrete number basis for the field, unlike the continuous amplitude basis in the QBM. This fact (which is true in the rotating wave and dipole approximation) implies that the 2LS plus EMF system is a resonant one. Hence even though the EM field has just as many (large number of) modes as the $\mathrm{HOB}$, only a very small fraction of them in a narrow range of the resonance frequency are efficiently coupled to the atom. This is the root cause for the very different qualitative behaviour between the QBM and the 2LS as far as decoherence is concerned.

One extreme case is that of a single mode field described by the Jaynes-Cummings model, where Rabi nutation takes place and the atom-field remains largely a coherent system. (For a coherent field, the probability for the atom to be found in the excited state at time $t$ regardless of the state of the field) obeys a Poisson distribution. This distribution in the photon number induces a spread in the Rabi frequencies, and causes collapse and revival of the Rabi nutation. These are distinct features of quantum coherence [1].) Adding all modes to the field we see spontaneous emission and the decay of the atom. The probability of an initially excited atom (remaining in the excited state) decays exponentially in the Wigner-Weisskopf form (characteristic of Markovian processes) with relaxation time constant $\Gamma$. For purely radiative decay the decay time $T_{1}$ of the inversion is half the decay time $T_{2}$ of the polarization. There is no large order of magnitude differences between dissipation and decoherence time (which in typical QBM high temperature conditions could be as high as 40 [19]). In fact it is perhaps inappropriate to talk about dissipation for a 2LA-EMF system because the conditions for a bath to actuate such a process is lacking. The transition from excited to ground state is closer in nature to relaxation (in the spin echo sense) than dissipation. In a cavity where excitation of the atom from the field (absorption) balances with emission, it is more appropriate to refer to the resonant state of the atom-field as a coherent system. In these senarios the distinction between QBM and 2LA cannot be clearer.

\section{Difference between $Q B M$ and $2 L S$}

So what led earlier authors to make the qualitative claim that 2LS decoheres easily? We think the confusion arises when the picture of QBM dissipation and decoherence is grafted on the 2LA-EMF system indiscriminantly. If the field which acts as the environment is a phonon field (from ion vibrations, see, e.g., [17]) and if the coupling is of the non-resonant type, then there is no disagreement. Decoherence should follow the QBM pattern as reported by many authors . Such sources (including atomic collisions in a cavity [7]) can be important for some setups. However, when one claims that the EM field can decohere a 2LS (with which it is coupled in a resonant way, as in the standard model) that is where we disagree.

Quantitatively, the model for the 2LS used by most authors for the discussion of decoherence inspired by QBM type of behavior has the atom in a $\sigma_{z}$ state (the diagonal Pauli matrix) coupled to the field mode operators $\hat{b}^{\dagger}, \hat{b}$. This type of coupling term (call it $\sigma_{z}$ type for convenience) commutes with the Hamiltonian of the system, and admits a diagonalization in the eigenbasis of the Hamiltonian. The field is coupled to the atom as a whole and thus is insensitive to the 2 level transition activity. In particular it does not probe the resonance or coherent properties of the two level atom, which is the most important feature, for quantum computation. By contrast the standard model for 2LA-EMF which we studied has a $\sigma_{ \pm}$coupling (call it standard coupling) to the field modes which highlights the 2 level activity of the atom and the field. This coupling considered in the standard model is indispensible, i.e., it cannot be removed from the two-level atom as it defines it and will be present in any realistic situation. What then is the origin of the QBM type of contribution to the 2LS?

If one accepts an environment other than the EM field, the question comes down to the characteristics of the experimental apparatus. For well- prepared ion traps we would expect it to be rather unimportant. If the EM field is the only field present, we can still ask if a QBM type of coupling term with the EM field would appear, and if yes, how strong would its effect be? This would be a useful way to accomodate the two different types of coupling terms.

Recall that the standard model is derived under the dipole and rotating wave approximation. In the next section we will show that the $\sigma_{z}$ type of coupling appears only in the next order expansion after the dipole approximation. Since these are good approximations for a large class of atomic states when the atom is nonrelativistic, the contribution from the QBM type of coupling used in [11, 12, 16] should be negligible and its ensuing decoherent effect insignificant. In this sense the EM field does not in leading order of approximation act like a bath in the QBM way, and coherence

\footnotetext{
${ }^{1}$ Even in such cases, one also needs to pay closer attention to the QBM behavior than what has been accorded for this model. Subtle points unnoticed before include, e.g., the imposition of a high frequency cutoff and Ohmic spectral function which restricts to a Markovian behavior 21] can lead to a violation of the positivity of the reduced density matrix [24], the violation of the fluctuation-dissipation relation [23], and the prolongation of coherence in a low temperature supraohmic bath [24]. They deserve more attention in the theoretical design of cavity qubit computers.
} 
in a 2LA-EMF system is quite well preserved (excepting other processes, e.g. [15,14]).

Our puzzle over the result on decoherence in the 2LS reported in the literature was what prompted us to begin this study. Without letting any familiar and convenient analogy influence our judgement, and without any preconceived notion, we choose to perform a first-principles calculation of the two-level atom (2LA)-electromagnetic field (EMF) system making as few assumptions and covering as wide a range of conditions as possible. We use the influence functional method [20] to take into account the full backreaction of the field on the atom, while adopting Grassmannian variables for the 2LA and the coherent state representation for the EMF. We find exact master equations for the full (non-Markovian) dynamics in the cases of a free quantum field and a cavity field at zero temperature.

In the next section we present the model and the formalism. A detailed derivation of our model is contained in Appendix A. In Sec. 3 we derive the master equations. In Sec. 4 we study different mode composition of the field, including that of an atom in a cavity. We end in Sec. 5 with a discussion of our findings and their implications.

This is the first in a series of papers on 2LAtom and quantum decoherence. The subsequent papers will treat 2LAEMF interaction at finite temperature, for EM fields in a coherent and squeezed state, and for multipolar models (where coupling other than the minimal is assumed). We will also tend to collective qubit systems and moving atoms interacting with an EM field. These results will have corresponding applications in atom optics and quantum computation problems.

\section{THE INFLUENCE FUNCTIONAL}

\section{A. The Model}

Our model for atom-field interaction is the standard one (see Appendix A for details) [1,2, 4, 2]. The total Hamiltonian for a (stationary) atom interacting with a quantum electromagnetic field (EMF) under the dipole, rotating wave (RW) and two-level (2L) approximation is given by

$$
\hat{H}=\hbar \omega_{0} \hat{S}_{z}+\hbar \sum_{\mathbf{k}}\left[\omega_{\mathbf{k}} \hat{b}_{\mathbf{k}}^{\dagger} \hat{b}_{\mathbf{k}}+\left(g_{\mathbf{k}} S_{+} \hat{b}_{\mathbf{k}}+\bar{g}_{\mathbf{k}} S_{-} \hat{b}_{\mathbf{k}}^{\dagger}\right)\right]
$$

where $\hat{b}_{\mathbf{k}}^{\dagger}, \hat{b}_{\mathbf{k}}$ are the creation and annihilation operators for the kth normal mode with frequency $\omega_{\mathbf{k}}$ of the electromagnetic field (thus for the field vacuum $\hat{b}_{\mathbf{k}}|0\rangle=0,\left[\hat{b}_{\mathbf{k}}, \hat{b}_{\mathbf{k}^{\prime}}^{\dagger}\right]=\delta_{\mathbf{k}, \mathbf{k}^{\prime}}$, for all $\mathbf{k}$.), and $\omega_{0}=\omega_{21}$ is the frequency between the two levels. Here

$$
\hat{S}_{z}=\frac{1}{2} \hat{\sigma}_{z}, \hat{S}_{ \pm}=\hat{\sigma}_{ \pm} \equiv \frac{1}{2}\left(\hat{\sigma}_{x} \pm i \hat{\sigma}_{y}\right)
$$

where $\sigma_{x, y, z}$ are the standard $2 \times 2$ Pauli matrices with $\sigma_{z}=\operatorname{diag}(1,-1)$, etc. The coupling constant $g_{\mathbf{k}} \equiv d_{21 \mathbf{k}} f_{\mathbf{k}}(\mathbf{X})$ where

$$
d_{i j \mathbf{k}} \equiv-\frac{i \omega_{i j}}{\sqrt{2 \hbar \omega_{\mathbf{k}} \epsilon_{0} V}} \mathbf{d}_{i j} \cdot \hat{\mathbf{e}}_{\mathbf{k} \sigma}
$$

and $\mathbf{d}_{i j} \equiv e \int \bar{\phi}_{i} \mathbf{x} \phi_{j} d^{3} x$ is the dipole matrix element between the eigenfunctions $\phi_{i}$ of the electron-field system, $\hat{\mathbf{e}}_{\mathbf{k} \sigma}$ is the unit polarization vector ( $\sigma=1,2$ are the two polarizations), and $f_{\mathbf{k}}(\mathbf{x})$ is the spatial mode functions of the vector potential of the electromagnetic field (in free space, $f_{\mathbf{k}}(\mathbf{x})=e^{-i \mathbf{k} \cdot \mathbf{x}}, V$ is the volume of space.). Under the dipole approximation $f_{\mathbf{k}}$ is evaluated at the position of the atom $\mathbf{X}$. Since $\mathbf{d}_{i j}=\overline{\mathbf{d}}_{j i}, \bar{d}_{i j \mathbf{k}}=d_{j i \mathbf{k}}$, we will choose a mode function representation such that $g_{\mathbf{k}}$ is real.

To see how this could possibly be related to the $\sigma_{z}$ type of coupling with Hamiltonian (used by e.g., 12 , 16 for the study of decoherence in 2LS)

$$
\hat{H}=\hbar \omega_{0} \hat{S}_{z}+\hbar \sum_{\mathbf{k}}\left[\omega_{\mathbf{k}} \hat{b}_{\mathbf{k}}^{\dagger} \hat{b}_{\mathbf{k}}+\hbar \sigma_{z}\left(\bar{g}_{\mathbf{k}} \hat{b}_{\mathbf{k}}+g_{\mathbf{k}} \hat{b}_{\mathbf{k}}^{\dagger}\right)\right]
$$

\footnotetext{
${ }^{2}$ Our Hamiltonian is given in the so-called minimal coupling (MC) as different from the multipolar coupling (MP) [6], which may be more relevant to atoms in a cavity because the expicit Coulomb interaction between the atom and its image charge is removed.
} 
we examine the next term after the dipole approximation in (A.15). This has a contribution to $g_{i j \mathbf{k}}$ even when $i=j$ which is equal to

$$
g_{i i \mathbf{k}}=c_{\mathbf{k}} \mathbf{k} \cdot \mathbf{q}_{i}
$$

where

$$
\mathbf{q}_{i}=\sum_{\sigma} \int \bar{\phi}_{i} \delta \mathbf{x}\left(\mathbf{p} \cdot \hat{\mathbf{e}}_{\mathbf{k} \sigma}\right) \phi_{i} d x^{3}
$$

and $c_{k}$ is a constant given by

$$
c_{\mathbf{k}}=-\frac{e}{m}\left(2 \hbar \omega_{\mathbf{k}} \epsilon_{0} V\right)^{-1 / 2}
$$

This generates an additional coupling term

$$
\sum_{\mathbf{k}} \sigma_{z}\left(g_{1 \mathbf{k}} b_{\mathbf{k}}+\bar{g}_{1 \mathbf{k}} b_{\mathbf{k}}^{\dagger}\right)+1\left(g_{2 \mathbf{k}} b_{\mathbf{k}}+\bar{g}_{2 \mathbf{k}} b_{\mathbf{k}}^{\dagger}\right)
$$

where

$$
g_{1 \mathbf{k}}=g_{11 \mathbf{k}}-g_{22 \mathbf{k}}, \quad g_{2 \mathbf{k}}=g_{11 \mathbf{k}}+g_{22 \mathbf{k}}
$$

This gives the lowest order $\sigma_{z}$ type of coupling in a 2LA -EMF system. The ratio of the coupling $g_{1 \mathbf{k}}$ of the $\sigma_{z}$ type in Eq. (II.3)to the dipole coupling $g_{\mathbf{k}}$ in Eq. (II.1) is

$$
\left|g_{1 \mathbf{k}} / g_{\mathbf{k}}\right|=\left|\frac{\mathbf{k}\left(\mathbf{q}_{1}-\mathbf{q}_{2}\right)}{m \omega_{\mathbf{k}} d_{12}}\right| \leq \frac{\omega_{\mathbf{k}}\left|\mathbf{q}_{1}-\mathbf{q}_{2}\right|}{m \omega_{\mathbf{k}} d_{12}}
$$

Thus the $\sigma_{z}$ type of coupling generated from the 2LA- EMF interaction will be significant only for very high frequencies $\omega_{k}$ of the EM field, a point intuitively clear from the meaning of the dipole approximation.

\section{B. Grassmannian Variables and Coherent State Integrals}

Since Feynman and Vernon 20] invented the influence functional method this formalism has been applied to treat the Brownian motion of a harmonic oscillator interacting with a harmonic oscillator bath by many authors 21, 9, 22, 24]. The two - level system in tunneling has been discussed in detail by Leggett et al [8], but the derivation of a master equation by this method which can traverse the non Markovian regimes has not yet been carried out. We shall perform such a calculation for a two level system, with the aid of Grassmaninn variables convenient for treating fermions, and the coherent state representation in a path integral form. We construct the coherent state of the combined atom-field system as

$$
|\{z\}, \eta\rangle=|\{z\}\rangle \times|\eta\rangle
$$

where $|z\rangle, z$ a complex number, denotes the EM field coherent states and $\mid \eta, \eta$ a Grassmannian or anticommuting number, denotes the electron coherent state. The transition amplitude between the initial state (i) at $t=0$ and the final state (f) at $t=t_{f}$ is expressed formally as [34] (here we suppress the index $k$,

$$
\left\langle\bar{\eta}_{f}, \bar{z}_{f} ; t \mid \eta_{i}, z_{i} ; 0\right\rangle=\int D z D \bar{z} D \eta D \bar{\eta} e^{\frac{i}{\hbar} S[z, \bar{z}, \eta, \bar{\eta}]}
$$

where the action is

$$
\frac{i}{\hbar} S[z, \bar{z}, \eta, \bar{\eta}]=\bar{z} z(t)+\bar{\eta} \eta(t)-\int_{0}^{t} d s\left(\bar{z} \dot{z}+\bar{\eta} \dot{\eta}+\frac{i}{\hbar} H(\bar{\eta}, \eta, \bar{z}, z)\right.
$$

Here $H$ is the Q-symbol of the Hamiltonian [35] and there is an implied summation over field modes. 


$$
H(\bar{\eta}, \eta, \bar{z}, z)=\hbar\left(\sum_{k} \omega_{k} \bar{z}_{k} z_{k}-g_{k}\left(\bar{z}_{k} \eta+\bar{\eta} z_{k}\right)+\omega_{0} \bar{\eta} \eta\right)
$$

In (II.8) we have substracted a constant term $\frac{1}{2} \omega_{0} 1$ to (II.1) so that the ground state now has zero energy. Henceforth we set $\hbar=1$.

The Hamiltonian in equation (2.8) is not a c-number function; it has terms that are odd. One might then question the validity of equation (2.6) for the path integral; it clearly exists as a formal expression, but its evaluation with a saddle point method, that is based on the Hamiltonian of equation (2.8) might be problematic. We dispell this doubt for the vacuum case with an operaytor method proof of the master equation in Appendix B. It shows that at least for the vacuum case tha saddle point evaluation yields the correct result. The general cases need separate considerations. For many qubits coupled to the EM field vacuum, we believe that the path integral method yields a simpler treatment than the operator method.

The integration is over all paths satisfying

$$
\begin{aligned}
z(0) & =z_{i} & \bar{z}(t) & =\bar{z}_{f} \\
\eta(0) & =\eta_{i} & \bar{\eta}(t) & =\bar{\eta}_{f}
\end{aligned}
$$

We assume initially that the density matrix of the total system+environment is factorizable $\hat{\rho}(0)=\hat{\rho}_{e}(0) \otimes \hat{\rho}_{b}(0)$. Only at that time would $z$ and $\eta$ be pure complex and Grassmannian numbers respectively. As the system evolves, both $\eta$ and $z$ contain Grassmann and c-number parts. The mixing of even and odd parts (note $g_{k}$ is odd) comes about as the initially factorized atom state becomes "dressed".

In the open system philosophy, as we are interested in the averaged effect of the field on the atom, the atom is considered as the 'system' while the field as the 'environment'. The path integral is performed over the variables $z$, while $\eta, \bar{\eta}$ are treated as external sources. When only one field mode is considered, we have

$$
\left\langle\bar{z}_{f} ; t \mid z_{i} ; 0\right\rangle_{\eta, \bar{\eta}}=\int D z D \bar{z} \exp \left\{\bar{z} z(t)-\int_{0}^{t} d s(\bar{z} \dot{z}+i(\omega \bar{z} z-g(\bar{z} \eta+\bar{\eta} z)(s)\}\right.
$$

with summation over paths satisfying the boundary condition (II.9 ) for $z$. We use the saddle point method. Minimizing the action yields the following equations

$$
\begin{aligned}
& \dot{z}+i \omega z=-i g \eta \\
& \dot{\bar{z}}-i \omega \bar{z}=i g \bar{\eta}
\end{aligned}
$$

with solutions

$$
\begin{aligned}
& z(s)=z_{i} e^{-i \omega s}-i g \int_{0}^{s} d s^{\prime} e^{-i \omega\left|s-s^{\prime}\right|} \eta\left(s^{\prime}\right) \\
& \bar{z}(s)=\bar{z}_{f} e^{-i \omega(t-s)}+i g \int_{s}^{t} d s^{\prime} e^{-i \omega\left|s-s^{\prime}\right|} \bar{\eta}\left(s^{\prime}\right)
\end{aligned}
$$

Using these for the transition amplitude (II.11 ) with the minimum value for the action, we obtain

$$
\begin{aligned}
& \left\langle\bar{z}_{f} ; t \mid z_{i} ; 0\right\rangle_{\eta, \bar{\eta}}=\exp \left\{\bar{z}_{f} z_{i} e^{-i \omega t}-i g\left[\bar{z}_{f} \int_{0}^{t} d s e^{-i \omega(t-s)} \eta(s)\right.\right. \\
& \left.\left.+\int_{0}^{s} d s e^{-i \omega s} \bar{\eta}(s) z_{i}\right]-g^{2} \int_{0}^{t} d s \int_{0}^{s} d s^{\prime} e^{-i \omega\left|s-s^{\prime}\right|} \bar{\eta}(s) \eta\left(s^{\prime}\right)\right\}
\end{aligned}
$$

A prefactor in the coherent state path integral is equal to one. Now the influence functional due to this single mode reads

$$
\begin{aligned}
\mathcal{F}\left[\eta, \bar{\eta} ; \eta^{\prime} \bar{\eta}^{\prime}\right]= & \int \frac{d \bar{z}_{i} d z_{i}}{\pi} \frac{d \bar{z}^{\prime}{ }_{i} d z_{i}^{\prime}}{\pi} \frac{d \bar{z}_{f} d z_{f}}{\pi} e^{-\bar{z}_{i} z_{i}-\bar{z}_{i}^{\prime} z_{i}^{\prime}-\bar{z}_{f} z_{f}} \\
& \times\left\langle\bar{z}_{f} ; t \mid z_{i} ; 0\right\rangle_{\eta, \bar{\eta}}\left\langle\bar{z}_{i}\left|\rho_{0}\right| z_{i}^{\prime}\right\rangle\left\langle\bar{z}_{i}^{\prime} ; 0 \mid z_{f} ; t\right\rangle_{\eta, \bar{\eta}}
\end{aligned}
$$

where the completeness relation for (unnormalized) coherent states has been used

$$
\int \frac{d \bar{z}_{i} d z_{i}}{\pi} e^{-\bar{z} z}|z\rangle\langle\bar{z}|=1
$$


Writing with an obvious identification

$$
\left\langle\bar{z}_{f} ; t \mid z_{i} ; 0\right\rangle_{\eta, \bar{\eta}}=\exp \left(A \bar{z}_{f} z_{i}+i \bar{z}_{f} \beta+i \bar{\gamma} z_{i}+D\right)
$$

we can use the identity

$$
\int \frac{d \bar{z}_{i} d z_{i}}{\pi} e^{-\bar{z} z+\bar{f} z+\bar{z} f}=e^{\bar{f} f}
$$

to obtain

$$
\mathcal{F}\left[\bar{\eta}, \eta, \bar{\eta}^{\prime}, \eta^{\prime}\right]=e^{\bar{\beta}^{\prime} \beta-\left(D+D^{\prime}\right)}
$$

for an initial vacuum state $\hat{\rho}_{0}=|0\rangle\langle 0|$. Substituting, we get the contribution to the influence functional from one mode

$$
\begin{aligned}
\mathcal{F}_{k}\left[\bar{\eta}, \eta, \bar{\eta}^{\prime}, \eta^{\prime}\right]= & \exp \left\{g _ { k } ^ { 2 } \int _ { 0 } ^ { t } d s \int _ { 0 } ^ { s } d s ^ { \prime } \left[\bar{\eta}^{\prime}(s) \eta\left(s^{\prime}\right) e^{-i \omega_{k}\left(s-s^{\prime}\right)}\right.\right. \\
& \left.\left.+\bar{\eta}^{\prime}\left(s^{\prime}\right) \eta(s) e^{i \omega_{k}\left(s-s^{\prime}\right)}-\bar{\eta}(s) \eta\left(s^{\prime}\right) e^{-i \omega_{k}\left(s-s^{\prime}\right)}-\bar{\eta}^{\prime}\left(s^{\prime}\right) \eta(s) e^{i \omega_{k}\left(s-s^{\prime}\right)}\right]\right\}
\end{aligned}
$$

The influence functional for all modes $\mathcal{F}=\prod_{k} \mathcal{F}_{k}$ is finally given by

$$
\begin{aligned}
\mathcal{F}\left[\bar{\eta}, \eta, \bar{\eta}^{\prime}, \eta^{\prime}\right]= & \exp \left\{\int _ { 0 } ^ { t } d s \int _ { 0 } ^ { s } d s ^ { \prime } \left(\mu\left(s-s^{\prime}\right)\left[\bar{\eta}^{\prime}(s)-\bar{\eta}(s)\right] \eta\left(s^{\prime}\right)\right.\right. \\
& \left.\left.+\mu^{*}\left(s-s^{\prime}\right) \bar{\eta}^{\prime}\left(s^{\prime}\right)\left[\eta(s)-\eta^{\prime}(s)\right]\right)\right\}
\end{aligned}
$$

in terms of the kernel

$$
\mu(s)=\sum_{k} g_{k}^{2} e^{-i \omega_{k} s}
$$

\section{THE MASTER EQUATION}

\section{A. The reduced density matrix propagator}

Having computed the influence functional we have an expression for the reduced density matrix propagator

$$
\begin{aligned}
J\left(\bar{\eta}_{f} \eta_{f}^{\prime} ; t \mid \bar{\eta}_{i}^{\prime} \eta_{i} ; 0\right)= & \int D \bar{\eta} D \eta D \overline{\eta^{\prime}} D \eta^{\prime} \exp \left\{\bar{\eta} \eta(t)+\bar{\eta}^{\prime} \eta^{\prime}(t)-\int_{0}^{t} d s\left(\bar{\eta} \dot{\eta}+\bar{\eta}^{\prime} \dot{\eta}^{\prime}+i \omega \bar{\eta} \eta-i \omega \bar{\eta}^{\prime} \eta^{\prime}(s)\right.\right. \\
& \left.+\int_{0}^{t} d s \int_{0}^{s} d s^{\prime}\left(\mu\left(s-s^{\prime}\right)\left[\bar{\eta}^{\prime}(s)+\bar{\eta}(s)\right] \eta\left(s^{\prime}\right)+\mu^{*}\left(s-s^{\prime}\right) \bar{\eta}^{\prime}\left(s^{\prime}\right)\left[\eta(s)+\eta^{\prime}(s)\right]\right)\right\}
\end{aligned}
$$

where the summation over all paths obey the boundary conditions (II.10) and

$$
\bar{\eta}^{\prime}(0)=\bar{\eta}_{i}^{\prime} \quad \eta^{\prime}(t)=\eta_{f}^{\prime}
$$

We can compute the path integral with saddle point evaluation and get

$$
\begin{array}{r}
\dot{\eta}+i \omega \eta+\int_{0}^{s} d s^{\prime} \mu\left(s-s^{\prime}\right) \eta\left(s^{\prime}\right)=0 \\
\dot{\bar{\eta}}^{\prime}-i \omega \bar{\eta}^{\prime}+\int_{0}^{s} d s^{\prime} \mu^{*}\left(s-s^{\prime}\right) \bar{\eta}^{\prime}\left(s^{\prime}\right)=0 \\
\dot{\eta}^{\prime}+i \omega \eta^{\prime}+\int_{0}^{s} d s^{\prime} \mu\left(s-s^{\prime}\right) \eta\left(s^{\prime}\right)-\int_{s}^{t} d s^{\prime} \mu^{*}\left(s-s^{\prime}\right)\left[\eta\left(s^{\prime}\right)+\eta^{\prime}\left(s^{\prime}\right)\right]=0 \\
\dot{\bar{\eta}}-i \omega \bar{\eta}+\int_{0}^{s} d s^{\prime} \mu^{*}\left(s-s^{\prime}\right) \bar{\eta}^{\prime}\left(s^{\prime}\right)-\int_{s}^{t} d s^{\prime} \mu\left(s-s^{\prime}\right)\left[\bar{\eta}^{\prime}\left(s^{\prime}\right)+\bar{\eta}\left(s^{\prime}\right)\right]=0
\end{array}
$$


It will turn out that only the solution of the first two of these equations will contribute to the path integral. We will therefore write

$$
\eta(s)=\eta_{i} u(s) \quad \bar{\eta}^{\prime}(s)=\bar{\eta}_{f}^{\prime} \bar{u}(s)
$$

where $u, \bar{u}$ are the solutions to equations (III.3),(III.4) under the condition

$$
u(0)=\bar{u}(t)=1
$$

Now equation (III.3) is a linear integrodifferential equation of first order and as such can be solved with the use of the Laplace transform and the convolution theorem. It is easy to show that

$$
u(s)=\mathcal{L}^{-1}\left(\frac{1}{z+i \omega+\tilde{\mu}(z)}\right)=\frac{1}{2 \pi i} \int_{c-i \infty}^{c+i \infty} \frac{d z e^{z s}}{z+i \omega+\tilde{\mu}(z)}
$$

where $\tilde{\mu}(z)$ is the Laplace transform of the kernel (II.24) and $c$ is a real constant larger than the real part of the poles of the integrand. It turns out that this function $u(s)$ contains all necessary information for the computation of the density matrix propagator. Substituting our expressions (III.8) and (III.9)) in (III.1) we can obtain the following expression for the propagator

$$
J\left(\bar{\eta}_{f} \eta_{f}^{\prime} ; t \mid \bar{\eta}_{i}^{\prime} \eta_{i} ; 0\right)=\exp \left(\bar{\eta}_{f} \eta_{i} u(t)+\bar{\eta}_{i}^{\prime} \eta_{f}^{\prime} \bar{u}(t)-[1-\bar{u}(t) u(t)] \bar{\eta}_{i}^{\prime} \eta_{i}\right)
$$

Since we are using coherent state path integrals we have departed in our evaluation from the standard saddle point approximation used on configuration space path integrals. In these cases, the standard procedure is to distinguish the imaginary part of the kernel as corresponding to dissipation and consider only its contribution when performing the saddle point evaluation. The resulting equations are then the classical dissipative equations of motion. But in the case of the coherent state path integral, there is no correspondence between extrema of the action and actual classical paths. Hence, there is no sense in splitting the kernel $\eta$ into real and imaginary part, and the saddle point evaluation should be carried out for the whole of the exponential.

\section{B. Master equation for a field in a vacuum state}

It is a standard procedure now to find the master equation 21,24. We compute the time derivative of the propagator:

$$
\dot{J}=\left(\dot{u} \bar{\eta}_{f} \eta_{i}+\dot{\bar{u}} \bar{\eta}_{i}^{\prime} \eta_{f}+\frac{d(\bar{u} u)}{d t} \bar{\eta}_{i}^{\prime} \eta_{i}\right) J
$$

The next step is to remove from the above equation the dependence on the initial values. This is done with the use of the following identities

$$
\eta_{i} J=\frac{1}{u} \frac{\delta J}{\delta \bar{\eta}_{f}} \quad \bar{\eta}_{i}^{\prime} J=\frac{1}{\bar{u}} \frac{\delta J}{\delta \eta_{f}^{\prime}}
$$

Note that we are suppressing (for ease of notation) symbols denoting left or right Grassmann differentiation. In all our expressions we implicitly assume that differentiation with respect to $\eta$ is always right and with respect to $\bar{\eta}$ always left.

For the density matrix at time $t$

$$
\rho_{t}\left(\bar{\eta}_{f}, \eta_{f}^{\prime}\right)=\int d \bar{\eta}_{i} d \eta_{i} e^{-\bar{\eta}_{i} \eta_{i}} d \bar{\eta}_{i}^{\prime} d \eta_{i}^{\prime} e^{-\bar{\eta}_{i}^{\prime} \eta_{i}^{\prime}} J\left(\bar{\eta}_{f} \eta_{f}^{\prime} ; t \mid \bar{\eta}_{i}^{\prime} \eta_{i} ; 0\right) \rho_{0}\left(\bar{\eta}_{i}, \eta_{i}^{\prime}\right)
$$

we obtain the evolution equation

$$
\frac{\partial}{\partial t} \rho=\frac{\dot{u}}{u} \bar{\eta} \frac{\delta \rho}{\delta \bar{\eta}}+\frac{\dot{\bar{u}}}{\bar{u}} \frac{\delta \rho}{\delta \eta} \eta+\frac{\frac{d}{d t}(\bar{u} u)}{\bar{u} u} \frac{\delta^{2} \rho}{\delta \eta \delta \bar{\eta}}
$$

This is one of our main results: The master equation for the two-level atom interacting with a an environment of electromagnetic field at its vacuum state. The effect of the field is contained within the function $u$ which can be 
determined by the solution of equation (III.3) or equivalently by the computation of the contour integral (III.9). In the next section we are going to find explicit expressions for $u$ for particular choices of the field configuration.

Let us return for the moment to equation (III.14) and write this in an operator language. It is easy to verify that:

$$
\bar{\eta} \frac{\delta \rho}{\delta \bar{\eta}}=\Sigma_{+} \rho \quad \frac{\delta \rho}{\delta \eta} \eta=\rho \Sigma_{+} \quad \frac{\delta^{2} \rho}{\delta \eta \delta \bar{\eta}}=S_{+} \rho S_{-}
$$

where $\Sigma_{+}=\left(1+\sigma_{z}\right) / 2$. If we write

$$
\frac{\dot{u}(t)}{u(t)}=\Gamma(t)+i \Omega(t)
$$

the master equation reads

$$
\frac{\partial}{\partial t} \rho=-i\left[\Omega(t) S_{+} S_{-}, \rho\right]+\Gamma(t)\left\{S_{+} S_{-}, \rho\right\}-2 \Gamma(t) S_{-} \rho S_{+}
$$

where

$$
H(t)=\Omega(t) \Sigma_{+}
$$

The first term corresponds to the unitary Hamiltonian evolution, only now the effect of the environment has induced a time dependent shift in the value of the frequency, the second term is time dependent dissipation and the third corresponds to noise.

\section{Spontaneous emission}

To show how the standard results are regained, and to understand the meaning of the new function in the master equation, let us consider the physical process of spontaneous emission. Start with a generic initial density matrix

$$
\rho=\left(\begin{array}{cc}
1-x & y \\
y^{*} & x
\end{array}\right)
$$

its corresponding Q-symbol is

$$
\rho(\bar{\eta}, \eta)=x+y^{*} \eta+y \bar{\eta}+(1-x) \bar{\eta} \eta
$$

If we evolve it with the density matrix propagator (III.10) we obtain for the state at time $t$

$$
\rho_{t}(\bar{\eta}, \eta)=1-\bar{u} u(1-x)+\left(\bar{u} y^{*} \eta\right)+(u \bar{\eta} y)+(\bar{u} u(1-x)) \bar{\eta} \eta
$$

corresponding to

$$
\rho_{t}=\left(\begin{array}{cl}
\bar{u} u(1-x) & u y \\
\bar{u} y^{*} & 1-\bar{u} u(1-x)
\end{array}\right)
$$

Considering the case $x=y=0$ we get for the probability of spontaneous emission

$$
P(1 \rightarrow 0, t)=1-\bar{u} u
$$

Also we should remark that the rate of decoherence in the energy eigenstates is governed by the absolute value of the function $u$ (the off- diagonal terms). But on the other hand $u$ itself determines the rate of nergy flow from the atom to the environment. Hence for our particular choice of initial state (vacuum) we find that decoherence and relaxation time are essentially identical. We shall use this equation to study decoherence in an ion trap in a later paper.

\section{FIELD MODES AND ANALYTIC U(T)}

Our master equation (III.17) depends solely on the function $u(t)$, which in its turn is determined by the kernel $\mu(s)$. In this section we will try to give some analytic expressions for this function in various different cases. 


\section{A. A single mode}

To connect with known results [1], let us start with the case when the field contains only a single mode with frequency $\omega_{k}=k$. Then $\mu(s)$ will read

$$
\mu(s)=g^{2} e^{-i k s}
$$

and

$$
\tilde{\mu}(z)=-g \int_{0}^{\infty} e^{-s z} e^{-i k s}=\frac{g^{2}}{z+i k}
$$

The integrand has two poles at the solutions of the equation

$$
z^{2}+i(\omega+k) z-\omega k+g^{2}=0
$$

given by

$$
z=-i \frac{\omega+k \pm\left[(\omega-k)^{2}+g^{2}\right]^{1 / 2}}{2}=-i \omega_{1,2}
$$

Hence

$$
u(s)=\frac{k-\omega_{1}}{\omega_{2}-\omega_{1}} e^{-i \omega_{1} s}-\frac{k-\omega_{2}}{\omega_{2}-\omega_{1}} e^{-i \omega_{2} s}
$$

This result is in agreement with standard ones [1]

\section{B. Infinite number of modes}

Now we consider the case of the vacuum electromagnetic field in free space i.e. not constrained by a cavity. The kernel will read then (using equation (II.24))

$$
\mu(s)=2 \lambda^{2} \int \frac{d^{3} k}{(2 \pi)^{3}} k^{-1} e^{-i k s}=\frac{\lambda^{2}}{\pi^{2}} \int_{0}^{\infty} k d k e^{-i k s}=\frac{d}{d s} \nu(s)
$$

where

$$
\nu(s)=\frac{i \lambda^{2}}{\pi^{2}} \int_{0}^{\infty} d k e^{-i k s}
$$

Note the factor of 2 in (IV.6) coming from the two photon polarisations and that we in view of (II.3) we have written $g_{\mathbf{k}}=\lambda \omega_{\mathbf{k}}^{-1 / 2}$.

Since the integral (IV.7) is not convergent, we will introduce an exponential cut-off in the higher frequency modes. The presence of the cut-off is of physical significance since we do not expect high electromagnetic modes to couple with our two-level atom.

Hence the kernel $\nu$ will read

$$
\nu(s)=\frac{i \lambda^{2}}{\pi^{2}} \int_{0}^{\infty} d k e^{-i k s-k \epsilon}=\frac{\lambda^{2}}{\pi^{2}} \frac{1}{s-i \epsilon}
$$

The Laplace transform of $\nu$ is then

$$
\tilde{\nu}(z)=\frac{\lambda^{2}}{\pi^{2}} \int_{0}^{\infty} d s \frac{e^{-s z}}{s-i \epsilon}=-\frac{\lambda^{2}}{\pi^{2}} e^{-i \epsilon z} E i(-i \epsilon z)
$$

where $E i$ denotes the exponential integral function analytically continued to the complex domain. At the limit $\epsilon \rightarrow 0$ this is essentially

$$
E i(-i \epsilon z)=\gamma+\log (-i \epsilon z)+O(\epsilon)
$$


where $\gamma$ is the Euler-Macheronni constant and the logarithm is taking values in the primary branch. Thus $\tilde{\mu}(z)$ reads $(\nu(0)$ is here $\nu(s=0)$ obtained by the integration by parts of the Laplace transform)

$$
\tilde{\mu}(z)=-\nu(0)+z \tilde{\nu}(z)=-\frac{i \lambda^{2}}{\pi^{2} \epsilon}-\frac{\lambda^{2}}{\pi^{2}} z e^{-i \epsilon z} E i(-i \epsilon z)
$$

Note that the cut-off $\epsilon$ affects significantly $\mu(z)$ only at large values of $z$, which essentially correspond to the very short time limit, i.e the time where the two-level atom starts "getting acquainted" with the photon reservoir. At larger times $(t>>\epsilon)$ we do not expect the cut-off to contribute significantly in the evolution. This is a rather typical behaviour in quantum Brownian motion models, provided that the ultraviolet cut - off of the environment is much larger than the natural frequencies of the system.

To evaluate the integral we first have to find the poles of the denominator. We can do that in a perturbation expansion . First let us absorb the divergent $\nu(0)$ factor in a frequency renormalisation.

$$
\tilde{\omega}=\omega-\frac{\lambda^{2}}{\pi^{2} \epsilon}
$$

so that we need find the zeros of the function $z+i \omega z-\frac{\lambda^{2}}{\pi^{2}} e^{-i \epsilon z} E i(-i \epsilon z)$. Looking for a solution in the vicinity of $z=-i \tilde{\omega}$ we find

$$
\begin{array}{r}
z=-i \tilde{\omega}-\tilde{\mu}(-i \tilde{\omega}) \\
-i\left(\tilde{\omega}-\frac{\lambda^{2} \tilde{\omega}}{\pi^{2}} \log \left(e^{\gamma} \epsilon \tilde{\omega}\right)\right)-\frac{\lambda^{2} \tilde{\omega}}{\pi}+O\left(\lambda^{4}\right):=-i \Omega-\Gamma
\end{array}
$$

We have a pole with a negative real part and we can verify numerically (also physically expected) that there is no pole with greater real part. This means when evaluating the inverse Laplace transform we can ignore the contribution of the branch - cut at $z=0$ (being on the right of the pole) and hence after some time, where all possible other poles with absolutely larger value of their real part will have stopped contributing the solution will be

$$
u(s)=e^{-i \Omega s-\Gamma s}
$$

This implies a Markovian time evolution and the identification of decoherence - relaxation time with $\Gamma^{-1}=\frac{\pi}{\lambda^{2} \omega}$. This is a general feature of the presence of a continuum of modes as can be seen from the case of an atom transparent to all modes but the ones in a strip, say $\left[\omega_{1}, \omega_{2}\right]$ containing the resonance frequency.

It is easy to verify that in this case we have again

$$
\tilde{\mu}(z)=-i \frac{\lambda^{2}}{\pi^{2}}\left(\omega_{2}-\omega_{1}\right)+\frac{\lambda^{2}}{\pi^{2}} \log \left(\frac{\omega_{2}-i z}{\omega_{1}-i z}\right)
$$

Hence defining again

$$
\tilde{\omega}=\omega-\frac{\lambda^{2}}{\pi^{2}}\left(\omega_{2}-\omega_{1}\right)
$$

we can find the pole at

$$
z=-i\left(\tilde{\omega}-\frac{\lambda^{2} \omega}{\pi^{2}} \log \left(\frac{\omega_{2}-\tilde{\omega}}{\tilde{\omega}-\omega-1}\right)\right)-\frac{\lambda^{2} \tilde{\omega}}{\pi}+O\left(\lambda^{4}\right)
$$

Note that the real part of the pole comes from the presence of a minus sign in a logarithm of some real valued object. Hence in the case where the atom 's frequency is outside the strip of interacting modes there will be no dissipation. This feature separates us from the QBM case, characterising the atom - field system as primarily a resonant one.

\section{Atom in a cavity}

Let us now consider the case of the atom lying within a cavity consisting of two parallel plates at distance $L$. The field satisfies Dirichlet boundary conditions on the surface of the plates, hence the modes in the normal direction to the plates are multiples of $\pi / L$. The kernel then reads 


$$
\begin{aligned}
\mu(s) & =\frac{\lambda^{2}}{2 \pi L} \sum_{n} \int_{0}^{\infty} \frac{k d k}{\left(k^{2}+(n \pi / L)^{2}\right)^{1 / 2}} e^{-i\left(k^{2}+(n \pi / L)^{2}\right)^{1 / 2}(s-i \epsilon)} \\
& =\frac{1}{2 \pi L} \sum_{n} \int_{|n \pi / L|}^{\infty} d k e^{-i k(s-i \epsilon)} \\
& =\frac{1}{2 \pi L} \frac{1}{\epsilon+i s} \sum_{n} e^{-i|n \pi / L|(s-i \epsilon)} \\
& =\frac{1}{2 \pi L} \frac{1}{\epsilon+i s} \frac{1+e^{-i \pi / L(s-i \epsilon)}}{1-e^{-i \pi / L(s-i \epsilon)}}
\end{aligned}
$$

Hence we can compute its Laplace transform

$$
\begin{aligned}
\tilde{\mu}(z) & =\frac{-i \lambda^{2}}{2 \pi L} \int_{0}^{\infty} \frac{d s e^{-s z}}{s-i \epsilon} \frac{1+e^{-i \pi / L(s-i \epsilon)}}{1-e^{-i \pi / L(s-i \epsilon)}} \\
& =\frac{-i \lambda^{2}}{2 \pi L} J\left(-i \epsilon z, \frac{i \pi}{2 L z}\right)
\end{aligned}
$$

where $J(x, a)$ is defined by

$$
J(x, a)=\int_{x}^{\infty} \frac{d y e^{-y}}{y} \operatorname{coth}(a y)
$$

and appears in equation (IV.20) through analytical continuation in the complex plane. This integral can actually be computed at the limit of vanishing $x(\epsilon \rightarrow 0)$ - see reference [37], equation 3.427.4.

$$
\begin{aligned}
\tilde{\mu}(z)= & \frac{-i \lambda^{2}}{\pi^{2} \epsilon}+\frac{\lambda^{2}}{\pi^{2}} z \log \left(i e^{\gamma} \epsilon z\right) \\
& -\frac{i \lambda^{2}}{\pi L}\left[\log \Gamma\left(\frac{L z}{i \pi}\right)-\frac{L z}{i \pi} \log \left(\frac{L z}{i \pi}\right)+\frac{L z}{i \pi}+\frac{1}{2} \log \frac{L z}{2 i \pi^{2}}\right]+O(\epsilon)
\end{aligned}
$$

Note that $\tilde{\mu}(z)$ is a sum of the term of case $2(L \rightarrow \infty)$ and a finite one (no dependence on $\epsilon$ ). The logarithm of the $\Gamma$-function gives a countable number of branch cuts at $z=-i \frac{n}{\pi L}, n$ positive integer, the resonance modes of the cavity. Again the important pole has a negative real part. We can again compute the pole perturbatively. It lies at

$$
\begin{aligned}
z= & -i\left(\tilde{\omega}+\frac{\lambda^{2}}{\pi^{2}} \tilde{\omega} \log \left(e^{\gamma} \epsilon \tilde{\omega}\right)-\frac{\lambda^{2}}{\pi L} \log \Gamma(-L \tilde{\omega} / \pi)\right. \\
& \left.-\frac{L \tilde{\omega}}{\pi} \log (-L \tilde{\omega} / \pi)-\frac{L \tilde{\omega}}{\pi}-\frac{1}{2} \log \left(-\frac{L \tilde{\omega}}{2 \pi^{2}}\right)\right)
\end{aligned}
$$

Clearly the logarithm of the gamma functions is the term out of which the real part of the pole appears. Since the real part of the pole is negative the branch cut is excluded from the integration contour and hence

$$
u(s)=e^{-i \Omega s-\Gamma s}
$$

Here $\Gamma$ gives a dissipation constant. . In Figure 1 we give a plot of the real part of the pole $(-\Gamma)$ as a function of the frequency $\omega$. Note that it has sharp maxima on the resonance points, implying persistence of coherence .

Already from the approximation (IV.23) we observe that the difference in the renormalized frequency from the case $L \rightarrow \infty(\Delta \omega)$ is finite. Unfortunately perturbation expansion is not reliable when $\tilde{\omega}$ is close to the resonance frequencies (this corresponds to negative integers arguments in the $\Gamma$-function where it diverges) and for this regime we have not been able to get any analytic results. In Figure 2 we have plotted the dependence of $\Delta \omega=\Omega[L]-\Omega[\infty]$ as the frequency changes. This effect of the frequency shift for an atom within the cavity is well known, as well as its relation to the Casimir effect [38]. 


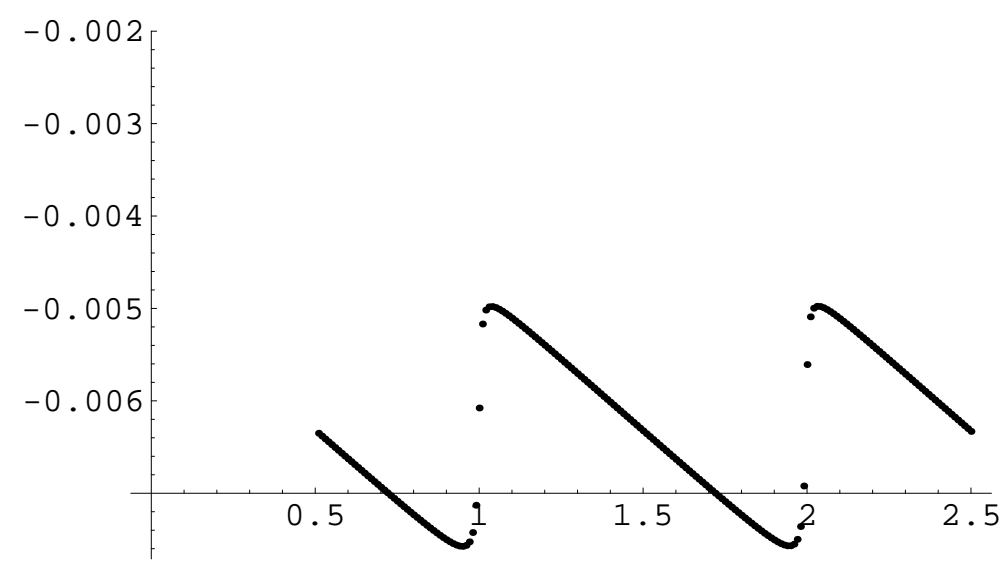

FIG. 1. Real part of pole (negative dissipation constant $\Gamma$ ) as a function of $\omega /(\pi / L)$.

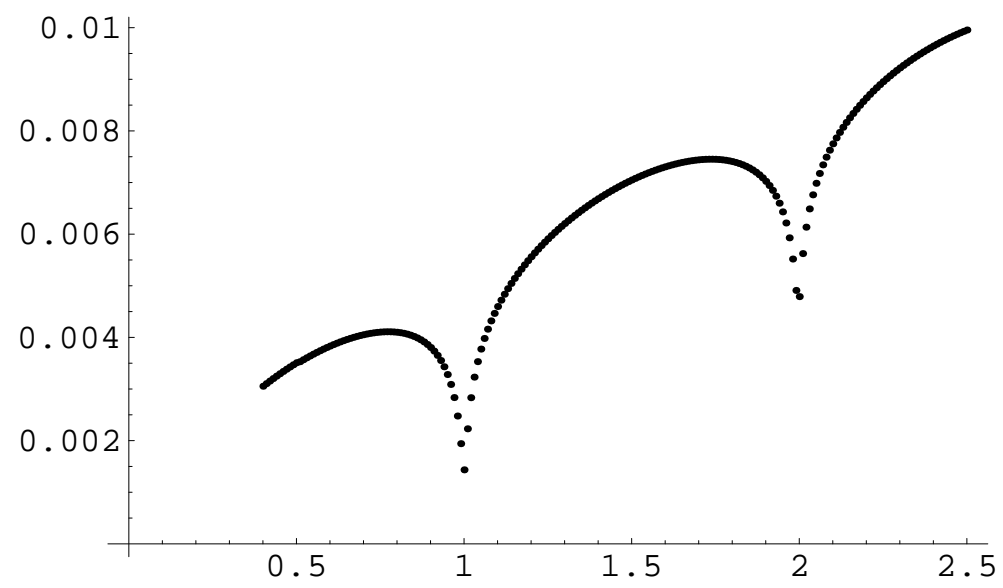

FIG. 2. Frequency shift $\Delta \Omega$ as a function of $\omega /(\pi / L)$.

\section{DISCUSSION}

Let us now integrate what we have found and look at the overall picture. The physics of a 2LA-EMF system at zero temperature is characterized by a number of time constants:

1) The inverse natural frequency $\omega_{0}^{-1}$

2) The inverse coupling constant $g_{k}^{-1}=\sqrt{\omega_{k}} / \lambda$

3) The relaxation time constant $\Gamma^{-1}$

4) The cavity size $L$ (divided by c)

First consider a zero temperature field in free space, thus ignoring factors 4). Start with only one mode in the field in resonance with the atom, then the system undergoes Rabi nutation with frequency $\Omega \approx g \sqrt{n+1}$, where $n$ is the photon number in the field. The collapse time (assuming a large mean photon number $\bar{n}$ ) is $g^{-1}$, and revival time is $2 \pi \sqrt{\bar{n}} / g$. [1]. Atom excitation becomes significant in a time much greater than $\omega_{0}^{-1}$ but shorter than $g^{-1}$. (This is the condition for a first order perturbation theory to give reasonable results.) For a large number of modes, spontaneous emission occurs at the relaxation time scale $\Gamma^{-1}=\pi / g>>\omega_{0}^{-1}$ which we found to be the same as the decoherence time - the time for the off diagonal elements of the reduced density matrix to decay (Sec. 4). When the mean number of photons in the field is large $(\bar{n}>>1)$, they become comparable to the collapse time. This is a measure of the coherence in the atom-field system, and is controlled mainly by their coupling and the photon number in the field. We see that with the resonance condition, the nature of decoherence in 2LS is very different from the QBM situation, where phase information in the Brownian particle is efficiently dispersed in the many modes in the bath coupled almost equally to the system. As we remarked in the Introduction, the identification of the phase information and energy flow from the 2LS to its environment is similar to the spin echo phenomena (Landau 'damping') which is 
based on statistical mixing rather than dissipation. The mathematical distinction lies between considering the system coupled to the discrete number basis (our model) and the continuous amplitude basis (QBM) of the environment. The latter case essentially produces noise that drives the system in a way insensitive to its own intrinsic dynamics. While in the former case, the coupling respects the internal dynamical structure of the 2LS and allows it to keep its coherence.

To see how the distribution of modes in a field changes the picture, the cavity field calculation in Sec. 4 is useful. As shown in Fig. 1, the relaxation constant develops peaks and minima. The resonance effect is enhanced by a cavity size commensurate with the natural frequency of the 2LA and dissipation weakens. Narrow band resonance fluorescence as well as inhibition of spontaneous decay by frequent measurements - the Quantum Zeno effect - are interesting phenomena which our equations can provide finer details.

Non-Markovian processes involve memory effects (nonlocal in time). For the QBM problem, except for the case of high temperature Ohmic bath which gives Markovian dynamics, other types of spectral density (supraohmic) or at low temperatures, the dynamics of the system is non-Markovian [24. When the reaction time of the bath is comparable to or faster than the natural time scale of the system $\left(\omega_{0}\right)$, one also expects to see non-Markovian behavior. By contrast, the $2 \mathrm{LA}$ is quite different: At zero temperature there is only one timescale $\Gamma^{-1}=\lambda^{-2} \omega^{-1}>>\omega^{-1}$ that determines both decoherence and relaxation. There is no memory effect and hence the process is Markovian. We expect that in finite temperature the dynamics of the 2LA will be nonMarkovian [39]. This is because there are more ways for the atom and the field to get entangled, and the memory effects of their interaction would presumably persist.

In conclusion we find that the 2LS interacting with an EM field is far more coherent than what is commonly believed, the misconception probably arising from the mistaken identification of this system with the Brownian model of an oscillator interacting with a harmonic oscillator bath.

Acknowledgement We wish to thank Phillip Johnson and Adrian Dragulescu for a close reading of our paper and making useful suggestions, and Sanjiv Shresta for checking the formulae and solutions to some nonlocal differential equations numerically and providing us with the figures. Sanjiv Shresta also first noticed a sign disrepancy in a sample calculation, which alerted us to possible ambiguities in the Grassmanian variable approach for finite temperature and coherent state fields. We also thank Dr Juan Pablo Paz for pointing out a mistake in equation (III.17) of an earlier version of this paper. This work is supported in part by NSF grant PHY98-00967. 


\section{APPENDIX A: ATOM-FIELD INTERACTION: TWO-LEVEL SYSTEM}

In this Appendix we give a rather detailed derivation of the Hamiltonian for a nonrelativistic atom interacting with a second-quantized electromagnetic field under the dipole, rotating wave and two level approximations. This is to facilitate the comparison of our model (II.1) with $\sigma_{ \pm}$coupling with that used by others (II.3) with $\sigma_{z}$ coupling. (See Introduction). To make this also useful for later papers in this series, we have included atomic motion. Note that the convention here is closer to [1] than that used in the text which is closer to [5]. The conversion is explained in footnote 2 .

The dynamics of a moving atom (mass $M$, momentum $\mathbf{P}$ ) whose electrons (charge $e$, mass $m$ ) interact with an electromagnetic field (vector potential $\mathbf{A}$, Coulomb potential $V$ ) is decribed by the (classical) Hamiltonian

$$
H=\frac{\mathbf{P}^{2}}{2 M}+\frac{1}{2 m}(\mathbf{p}-e \mathbf{A})^{2}+e V(\mathbf{x})+H_{b}
$$

where $H_{b}$ is the Hamiltonian for the electromagnetic field. Expanding out one can write this as

$$
H=H_{a}+H_{e}+H_{b}+H_{c}
$$

where

$$
H_{a}=\frac{\mathbf{P}^{2}}{2 M}
$$

describes nonrelativistic atom motion,

$$
H_{e}=\frac{p^{2}}{2 m}+e V(\mathbf{x})
$$

decribes the dynamics of the (one) electron, while

$$
H_{c 1}=-\frac{e}{m} \mathbf{A} \cdot \mathbf{p}
$$

and

$$
H_{c 2}=\frac{e^{2}}{2 m} \mathbf{A}^{2}
$$

describe the coupling between the electron and the field. The second term makes no contribution to one-photon processes and will be ignored. We will refer to $H_{0}=H_{e}+H_{b}$ as the unperturbed Hamiltonian, and $H_{I}=H_{c 1}$ the interaction Hamiltonian.

In a second-quantized form, the Hamiltonian for the radiation field is given by

$$
\hat{H}_{b}=\sum_{\mathbf{k}} \hbar \omega_{\mathbf{k}} \hat{b}_{\mathbf{k}}^{\dagger} \hat{b}_{\mathbf{k}}
$$

where $\hat{b}_{\mathbf{k}}^{\dagger}, \hat{b}_{\mathbf{k}}$ are the creation and annihilation operators for the kth normal mode of a free massless vector field. Thus for the field vacuum $b_{\mathbf{k}}|0\rangle=0, \hat{b}_{\mathbf{k}}|0\rangle=0,\left[\hat{b}_{\mathbf{k}}, \hat{b}_{\mathbf{k}^{\prime}}^{\dagger}\right]=\delta_{\mathbf{k}, \mathbf{k}^{\prime}}$, for all $\mathbf{k}$. We can perform a harmonic decomposition of the vector potential of the electromagnetic field

$$
\mathbf{A}(\mathbf{x}, t)=\sum_{\mathbf{k}}\left(\frac{\hbar}{2 \omega_{\mathbf{k}} \epsilon_{0}}\right)^{\frac{1}{2}}\left[b_{\mathbf{k} \sigma} \mathbf{u}_{\mathbf{k} \sigma}(\mathbf{x}) e^{i \omega_{\mathbf{k}} t}+b_{\mathbf{k} \sigma}^{\dagger} \overline{\mathbf{u}}_{\mathbf{k} \sigma}(\mathbf{x}) e^{-i \omega_{\mathbf{k}} t}\right]
$$

where, assuming the field is contained in a box of size L, the spatial mode functions $\mathbf{u}_{\mathbf{k} \sigma}$ is given by

$$
\mathbf{u}_{\mathbf{k} \sigma}(\mathbf{x})=L^{-3 / 2} \hat{\mathbf{e}}_{\mathbf{k} \sigma} f_{\mathbf{k}}(\mathbf{x})
$$

Here $\hat{\mathbf{e}}_{\mathbf{k} \sigma}$ is the unit polarization vector and $\sigma=1,2$ are the two (transverse) polarizations. In free space, $f_{\mathbf{k}}(\mathbf{x})=$ $e^{-i \mathbf{k} \cdot \mathbf{x}}$.

We assume that electron motion is much faster than the motion of the atom, thus it sees a stationary central Coulomb potential around the center of mass of the atom. Denoting the (time-independent, nonrelativistic) electronic 
wave function eigenstate belonging to the eigenvalue $E_{i}$ by $\phi_{i}$, i.e, $H_{e} \phi_{i}=E_{i} \phi_{i}$, we can write the Hamiltonian for the electron in the second-quantized form as

$$
\hat{H}_{e}=\sum_{i} E_{i} \hat{a}_{i}^{\dagger} \hat{a}_{i}
$$

where $i$ labels the bound states of the eletron (we assume vanishing probability for the atom to ionize) and $\hat{a}_{i}^{\dagger}$ and $\hat{a}_{i}$ are the creation and annihilation operators. As fermions they obey the anticommutation relations $\hat{a}_{i}^{\dagger} \hat{a}_{j}+\hat{a}_{j} \hat{a}_{i}^{\dagger}=\delta_{i j}$.

To perform perturbation theory, the electronic wave function of the interacting system is expanded in terms of the eigenfunctions $\phi_{i}$ of the unperturbed Hamiltonian, with basis formed by the direct product of the electron and the field states Thus the electron field operator $\hat{\psi}(\mathbf{x})$ can be expanded as

$$
\hat{\psi}(\mathbf{x})=\sum_{i} \hat{a}_{i} \phi_{i}(\mathbf{x})
$$

With this, the interaction Hamiltonians is given by

$$
\hat{H}_{I}=-\frac{e}{m} \int \hat{\psi}^{\dagger}(\mathbf{x})(\mathbf{p} \cdot \mathbf{A}) \hat{\psi}(\mathbf{x}) d^{3} x
$$

or in terms of $\hat{a}_{i}, \hat{b}_{\mathbf{k}}$ operators

$$
\hat{H}_{I}=\hbar \sum_{i, j, \mathbf{k}} \hat{a}_{i}^{\dagger} \hat{a}_{j}\left(g_{i j \mathbf{k}} \hat{b}_{\mathbf{k}}+\bar{g}_{i j \mathbf{k}} \hat{b}_{\mathbf{k}}^{\dagger}\right)
$$

where

$$
g_{i j \mathbf{k}}=-\frac{e}{m} \frac{1}{\sqrt{2 \hbar \omega_{\mathbf{k}} \epsilon_{0}}} \int \bar{\phi}_{i}(\mathbf{x}) \mathbf{u}_{\mathbf{k} \sigma}(\mathbf{x}) \cdot \mathbf{p} \phi_{j}(\mathbf{x}) d^{3} x
$$

\section{Dipole Approximation}

Now consider conditions when the spatial variation of the vector potential $\mathbf{A}$ of the electromagnetic field is small compared to the electronic wave function $\psi$, one can expand $f_{\mathbf{k}}(\mathbf{x})$ in $\mathbf{u}_{\mathbf{k} \sigma}(\mathbf{x})$ around the position of the atom $\mathbf{x}=\mathbf{X}+\delta \mathbf{x}:$

$$
e^{i \mathbf{k} \cdot \mathbf{x}}=e^{i \mathbf{k} \cdot \mathbf{X}}\left[1+\mathbf{k} \cdot \delta \mathbf{x}-\frac{1}{2}(\mathbf{k} \cdot \delta \mathbf{x})^{2}+\ldots\right]
$$

The dipole approximation amounts to keeping just the leading term. Doing so, we can take the field mode function $f_{\mathbf{k}}(\mathbf{x})$ out of the integration above and evaluate it at the atomic position. To evaluate

$$
\frac{e}{m} \int \bar{\phi}_{i} \mathbf{p} \phi_{j} d^{3} x
$$

we make use of

$$
\frac{d \hat{x}_{i}}{d t}=\frac{\hat{p}_{i}}{m}=\frac{1}{i \hbar}\left[\hat{x}_{i}, \hat{H}_{e}\right]
$$

yielding,

$$
\frac{e}{m} \int \bar{\phi}_{i} \mathbf{p} \phi_{j} d^{3} x=i \omega_{i j} \mathbf{p} d_{i j}
$$

where $\hbar \omega_{i j}=E_{i}-E_{j}$, and $\mathbf{d}_{i j} \equiv e \int \bar{\phi}_{i} \mathbf{x} \phi_{j} d^{3} x$ is the dipole matrix element, $\mathbf{d}_{i j}=\overline{\mathbf{d}}_{j i}$. Define

$$
d_{i j \mathbf{k}} \equiv-\frac{i \omega_{i j}}{\sqrt{2 \hbar \omega_{\mathbf{k}} \epsilon_{0} V}} \mathbf{d}_{i j} \cdot \hat{\mathbf{e}}_{\mathbf{k} \sigma}
$$

Note that $\bar{d}_{i j \mathbf{k}}=d_{j i \mathbf{k}}$. With this under the dipole approximation, 


$$
g_{i j \mathbf{k}}=d_{i j \mathbf{k}} f_{\mathbf{k}}(\mathbf{X})
$$

\section{Rotating Wave Approximation}

In the interaction picture, recalling that the time evolution of the ladder operators are given by

$$
\hat{a}_{i}^{\dagger}(t)=\hat{a}_{i}^{\dagger} e^{i \omega_{i} t} \quad \hat{a}_{j}(t)=\hat{a}_{j} e^{-i \omega_{j} t} \quad \text { and } \quad \hat{b}_{\mathbf{k}}(t)=\hat{b}_{\mathbf{k}} e^{-i \omega_{\mathbf{k}} t}
$$

the interaction Hamiltonian $H_{I}$ in the interaction picture becomes

$$
\tilde{H}_{I}=\hbar \sum_{i, j, \mathbf{k}} g_{i j \mathbf{k}} \hat{a}_{i}^{\dagger} \hat{a}_{j} \hat{b}_{\mathbf{k}} e^{i\left(\omega_{i j}-\omega_{\mathbf{k}}\right)}+\hbar \sum_{i j \mathbf{k}} \bar{g}_{i j \mathbf{k}} \hat{a}_{i}^{\dagger} \hat{a}_{j} \hat{b}_{\mathbf{k}}^{\dagger} e^{i\left(\omega_{i j}+\omega_{\mathbf{k}}\right)}
$$

where $\omega_{i j} \equiv \omega_{i}-\omega_{j}$. We see there are two types of oscillatory terms present: $e^{-i\left(\omega_{i j} \pm \omega_{\mathbf{k}}\right)}$. Processes most effective in the absorption or emission of a photon by the atom correspond to those with near resonance frequency $\omega_{i j} \approx \omega_{\mathbf{k}}$. Assuming $\omega_{i j}>0\left(E_{i}>E_{j}\right)$, the first type with $e^{-i\left(\omega_{i j}+\omega_{\mathbf{k}}\right)}$ has a rapidly oscillating phase and its contribution is small compared with the second type with $e^{-i\left(\omega_{i j}-\omega_{\mathbf{k}}\right)}$ whose stationary phase at near resonance gives a large contribution. Physically the first type corresponds to either the excitation of the atom along with the emission of a photon or the relaxation of the atom along with the absorption of a photon, which is less probable than the second type corresponding to the excitation of an atom upon the absorpsion of a photon or the relaxation of an atom with the emission of a photon. We shall therefore ignore the first type of terms, which amounts to working under the Rotating Wave Approximation. This is the second major approximation in this standard model.

\section{Two-Level Atom}

Let us now consider the idealized case when the atom has only two electronic states, $|+>|-,>$ corresponding to $i=2,1$, with energies equal to $E_{ \pm}= \pm \frac{1}{2} \hbar \omega_{0}$. (The two states can interchangeably be labeled as $|1>| 0>$,, or $|e>| g>$, or $|\uparrow>,| \downarrow>$.) Thus $\omega_{i j=21}=\omega_{0}$. Thus

$$
\hat{H}_{a}=\frac{\hbar \omega_{0}}{2}\left(a_{2}^{\dagger} a_{2}-a_{1}^{\dagger} a_{1}\right) \equiv \frac{\hbar \omega_{0}}{2} \sigma_{z} \equiv \hbar \omega_{0} S_{z}
$$

where we have introduced a Pauli matrix $(2 \times 2)$ representation $\sigma_{z}=\operatorname{diag}(1,-1)$ For the interaction Hamiltonian above, under the RWA, in the $i=2, j=1$ contribution to the summation, the first line containing $e^{i\left(\omega_{0}-\omega_{\mathbf{k}}\right)}$ is kept, while the second line is dropped. The reverse is true for the $i=1, j=2$ term. The interaction Hamiltonian (in the interaction picture) now becomes

$$
\tilde{H}_{I}=\hbar \sum_{\mathbf{k}}\left[g_{21 \mathbf{k}} \hat{a}_{2}^{\dagger} \hat{a}_{1} \hat{b}_{\mathbf{k}} e^{-i\left(\omega_{\mathbf{k}}-\omega_{21}\right) t}+\bar{g}_{12 \mathbf{k}} \hat{a}_{1}^{\dagger} \hat{a}_{2} \hat{b}_{\mathbf{k}}^{\dagger} e^{-i\left(\omega_{\mathbf{k}}+\omega_{12}\right) t}\right]
$$

Introducing the Pauli matrix representation for the fermion operators $a_{2}^{\dagger} a_{1} \rightarrow \sigma_{+} \equiv S_{+}$and $a_{1}^{\dagger} a_{2} \rightarrow \sigma_{-} \equiv S_{-}$, and defining $g_{\mathbf{k}} \equiv d_{21 \mathbf{k}}=\bar{d}_{12 \mathbf{k}}$ (recall $g_{i j \mathbf{k}} \equiv d_{i j k} f_{\mathbf{k}}(\mathbf{X})$ ) we can write the interaction Hamiltonian (in the Heisenberg picture) in a simple form:

$$
\left.\hat{H}_{I}=\hbar \sum_{\mathbf{k}} g_{\mathbf{k}}\left(S_{+} b_{\mathbf{k}} f_{\mathbf{k}}(\mathbf{X})+S_{-} b_{\mathbf{k}}^{\dagger} \bar{f}_{\mathbf{k}}(\mathbf{X})\right)\right)
$$

Therefore the total Hamiltonian for our model of a moving atom interacting with a quantum electromagnetic field under the dipole, rotating wave and two-level approximation is given by

$$
\hat{H}=\frac{\hat{\mathbf{P}}^{2}}{2 M}+\hbar \omega_{0} \hat{S}_{z}+\hbar \sum_{\mathbf{k}}\left[\omega_{\mathbf{k}} \hat{b}_{\mathbf{k}}^{\dagger} \hat{b}_{\mathbf{k}}+g_{\mathbf{k}}\left(f_{\mathbf{k}}(\mathbf{X}) S_{+} b_{\mathbf{k}}+\bar{f}_{\mathbf{k}}(\mathbf{X}) S_{-} b_{\mathbf{k}}^{\dagger}\right)\right]
$$




\section{APPENDIX B: AN OPERATOR PROOF OF THE MASTER EQUATION}

We use the resolvent decomposition of the propagator

$$
e^{-i H t}=\int \frac{d E e^{-i E t}}{E-H}
$$

Then by writing $H=H_{0}+H_{I}$ we can expand the resolvent and get

$$
\begin{array}{r}
(E-H)^{-1}=\left(E-H_{0}\right)^{-1}\left(1-\left(E-H_{0}\right)^{-1} H_{I}\right)^{-1} \\
=\left(E-H_{0}\right)^{-1}\left(1+\left(E-H_{0}\right)^{-1} H_{I}+\frac{1}{2}\left(E-H_{0}\right)^{-1} H_{I}\left(E-H_{0}\right)^{-1} H_{I}+\ldots\right)
\end{array}
$$

When we act the expanded resolvent in any vector $|0, i\rangle=|0\rangle \otimes|i\rangle,(|i\rangle$ denotes an eigenstate of the Hamiltonian of the two level system) we see that

only expanded terms that contain alternating sequences of $\sigma_{+}$and $\sigma_{-}$survive. This makes the summation much easier. Also if we note that

$$
\begin{aligned}
& \left(E-H_{0}\right)^{-1} \sum_{k} g_{k} b_{k}\left(E-H_{0}\right)^{-1} g_{k^{\prime}} \sum_{k^{\prime}} b_{k^{\prime}}^{\dagger}|0\rangle \\
= & \left(E-\omega_{0}\right)^{-1} \sum_{k} \frac{g_{k}^{2}}{E-\omega_{k}}|0\rangle:=\left(E-\omega_{0}\right)^{-1} F(E)
\end{aligned}
$$

Hence we can compute the matrix elements by resumming the expansion

$$
\begin{aligned}
\left\langle z, 0\left|(E-H)^{-1}\right| 0,0\right\rangle & =E^{-1} \\
\left\langle z, 1\left|(E-H)^{-1}\right| 0,0\right\rangle & =0 \\
\left\langle z, 1\left|(E-H)^{-1}\right| 0,1\right\rangle & =\left(E-\omega_{0}-F(E)\right)^{-1} \\
\left\langle z, 0\left|(E-H)^{-1}\right| 0,0\right\rangle & =\frac{E-\omega_{0}}{E-\omega_{0}-F(E)} \sum_{k} \frac{g_{k}}{(E-\omega)^{2}} \bar{z}_{k}
\end{aligned}
$$
is

The reduced density matrix propagator in the energy basis of the two- level atom and for the vacuum initial state

$$
\begin{array}{r}
J(i, j ; t \mid m, n ; 0)=\int d E d E^{\prime} e^{-i\left(E-E^{\prime}\right) t} \int D z D \bar{z} e^{-\sum_{k} \bar{z}_{k} z_{k}} \\
\left(\left\langle z, i\left|(E-H)^{-1}\right| 0, m\right\rangle\left\langle 0, n\left|(E-H)^{-1}\right| z, j\right\rangle\right)
\end{array}
$$

We can then verify that the only nonzero elements $m n \rightarrow i j$ are the following and their conjugates

$$
\begin{array}{r}
J(0,0 ; t \mid 0,0 ; 0) \rightarrow E^{-1} E^{\prime-1} \\
J(0,1 ; t \mid 0,1 ; 0) \rightarrow E^{-1}\left(E^{\prime}-\omega_{0}-F\left(E^{\prime}\right)\right)^{-1} \\
J(0,0 ; t \mid 0,0 ; 0) \rightarrow \frac{E-\omega_{0}}{E-\omega_{0}-F(E)} \frac{E^{\prime}-\omega_{0}}{E^{\prime}-\omega-F\left(E^{\prime}\right)} \sum_{k} \frac{g_{k}^{2}}{\left(E-\omega_{k}\right)\left(E^{\prime}-\omega_{k}\right)} \\
J(11 ; t \mid 1,1 ; 0) \rightarrow\left(E-\omega_{0}-F(E)\right)^{-1}\left(E-\omega_{0}-F\left(E^{\prime}\right)\right)^{-1}
\end{array}
$$

Then it is easy to check that this reproduces the propagation as given by equation (3.21) that was obtained through the influence functional method. Indeed

$$
u(t)=\int \frac{d E e^{-i E t}}{E-\omega_{0}-F(E)}
$$

is exactly the same as the one defined by equation (3.9). 
[1] D. F. Walls and G. J. Milburn, Quantum Optics ( Springer Verlag, Berlin, Heidelberg, 1994).

[2] L. Mandel and E. Wolf, Optical Coherence and Quantum Optics (Cambridge University Press, Cambridge, 1995).

[3] O. Scully and M. Suhail Zubairy, Quantum Optics (Cambridge University Press, Cambridge, 1997).

[4] M. Weissbluth, Photon-Atom Interactions (Academic Press, San Diego, 1988).

[5] W. Vogel and D. G. Welsch, Lectures on Quantum Optics, (Akademie Verlag, Berlin, 1994).

[6] G. Compagno, R. Passante and F. Persico, Atom-Field Interactions and Dressed Atom (Cambridge University, Cambridge, 1995).

[7] H. J. Carmichael, An Open Systems Approach to Quantum Optics (Springer Verlag, Berlin, 1993).

[8] A. O. Caldeira and A. J. Leggett, Ann. Phys. (NY) 149, 374 (1983). A. J. Leggett et al., Rev. Mod. Phys. 59, 1 (1987).

[9] U. Weiss, Quantum Dissipative Systems (World Scientific, Singapore, 1993).

[10] I. L. Chuang et al, Science 270, 1633 (1995); D. P. Di Vencenzo, Science 270, 255 (1995). P. W. Shor, Phys. Rev. A52, R2493 (1995). J. I. Cirac and P. Zoller, Phys. Rev. Lett. 74, 4091 (1995). A. Ekert and R. Jozsa, Rev. Mod. Phys. 68, 733 (1996).

[11] W. G. Unruh, Phys. Rev. A51, 992 (1995).

[12] G. M. Palma, K.-A. Suominen and A. K. Ekert, Proc. Roy. Soc. London A 452, 567 (1996).

[13] P. Zanardi and M. Rasetti, Phys. Rev. Lett. 79, 3306 (1997).

[14] M. B. Plenio and P. L. Knight, Proc. Rey. Soc. Lon. A453, 2017 (1997).

[15] S. Schneider and G. J. Milburn, quant-ph/9710044 (1997).

[16] L. Viola and S. Lloyd, Phys. Rev. A58, 2733 (1998).

[17] A. Garg, quant-ph/9803071 (1998).

[18] P. Zanardi , Phys. Rev. A57, 3276 (1998).

[19] See, e.g., W. H. Zurek, Physics Today 44, 36 (1991); Prog. Theor. Phys. 89, 281 (1993). E. Joos and H. D. Zeh, Z. Phys. B59, 223 (1985); D. Giulini et al, Decoherence and the Appearance of a Classical World in Quantum Theory (Springer Verlag, Berlin, 1996).

[20] R. P. Feynman and A. R. Hibbs, Quantum Mechanics and path integrals (McGraw-Hill, New York, 1965) ; R. P. Feynman and F. L. Vernon, Ann. Phys. (N. Y.) 24, 118 (1963).

[21] A. O. Caldeira and A. J. Leggett, Physica A 121, 587 (1983). Phys. Rev. A31, 1059 (1985).

[22] H. Grabert, P. Schramm and G. L. Ingold, Phys. Rep. 168, 115 (1988).

[23] W. G. Unruh and W. H. Zurek, Phys. Rev. 40, 1071 (1989).

[24] B. L. Hu, H. P. Paz and Y. Zhang, Phys. Rev. D45, 2843 (1992); Phys. Rev. D47, 1576 (1993).

[25] J. P. Paz, S. Habib and W. H. Zurek, Phys. Rev. 47, 488 (1993).

[26] B. L. Hu and A. Matacz, Phys. Rev. D 49, 6612 (1994).

[27] B. L. Hu and Y. Zhang, Mod. Phys. Lett. A8, 3575 (1993), Int. J. Mod. Phys. 10, 4537 (1995).

[28] A. Anderson and J. J. Halliwell, Phys. Rev. D48, 2753 (1993); C. Anastopoulos and J. J. Halliwell, Phys. Rev. D51, 6870 (1995); C. Anastopoulos, Phys. Rev. E53, 4711 (1996).

[29] J. R. Anglin, R. Laflamme, W. H. Zurek and J. P. Paz, Phys. Rev. D52, 2221 (1995).

[30] B. L. Hu and D. Pavon, Phys. Lett. B180, 329 (1986); H. E. Kandrup, Phys. Rev. D37, 3505 (1988); D38, 1773 (1988).

[31] S. Habib, Y. Kluger, E. Mottola and J. P. Paz, Phys. Rev. Lett. 76, 4660 (1996)

[32] S. K. Ma, Statistical Mechanics (World Scientific, Singapore, 1995)

[33] P. M. V. Barone and A. O. Caldeira, Phys. Rev. A 43, 57 (1991).

[34] See, e.g., J. W. Negel and H. Orland, Quantum Many-Particle Systems (Addison-Wesley, 1988)

[35] T. W. B. Kibble, Lectures in Quantum Field Theory, (unpublished).

[36] B. Davies, Integral Transforms and their Applications, Springer Verlag, New York (1978).

[37] I.S. Gradshteyn and I. M. Ryzhik, Table of Integrals, Series and Products, Academic Press, Orlando (1965).

[38] G. Barton, Phys. Rept. 170, 1 (1988); 170, 65 (1988).

[39] Charis Anastopoulos, Adrian Dragulescu, B. L. Hu and Sanjiv Shresta, "Two-Level Atom-Field Interaction at Finite Temperatures" 